\title{
A note on major sequences and external activity in trees
}

\author{
Janet S. Beissinger \\ Institute for Mathematics and Science Education \\ The University of Illinois at Chicago (M/C 250) \\ 950 S. Halsted Street, Chicago, IL 60607-7019 \\ Beissing@uic.edu
}

and

Uri N. Peled

Dept. of Mathematics, Statistics, and Computer Science

The University of Illinois at Chicago (M/C 249)

851 S. Morgan Street, Chicago, IL 60607-7045

uripeled@uic.edu

Submitted: September 20, 1996; Accepted October 31, 1996.

\begin{abstract}
A bijection is given from major sequences of length $n$ (a variant of parking functions) to trees on $\{0, \ldots, n\}$ that maps a sequence with sum $\left(\begin{array}{c}n+1 \\ 2\end{array}\right)+k$ to a tree with external activity $k$.

Key Words: Major sequence, external activity, parking function, bijection

Mathematical Reviews Subject Numbers: Primary 05A19; Secondary 05A15, 05C05, 05C30
\end{abstract}


We present a bijection from major seqeunces (a variant of parking funtions) of length $n$ to trees on $\{0, \ldots, n\}$ that takes area to external activity. Our main tool is a decomposition of major sequences due to Kreweras [6].

An integer sequence $S=\left(s_{1}, \ldots, s_{n}\right)$ is called a major sequence of length $n$ [6] if its non-decreasing rearrangement $\left(z_{1}, \ldots, z_{n}\right)$ satisfies

$$
z_{i} \geq i \quad \text { for all } \quad 1 \leq i \leq n \text { and } z_{n} \leq n
$$

Another way to view $\left(z_{1}, \ldots, z_{n}\right)$ is as a lattice path from $(0,0)$ to $(n, n)$ that never drops below the main diagonal. In Figure 1 the top lattice path represents the nondecreasing rearrangement of the major sequence

$$
(7,8,8,3,3,5,3,7)
$$

and the bottom represents the identity

$$
(1,2,3,4,5,6,7,8)
$$



Figure 1: The nondecreasing rearrangement of the major sequence $(7,8,8,3,3,5,3,7)$ with length 8 and area 8.

We note that $\left(s_{1}, \ldots, s_{n}\right)$ is a major sequence iff $\left(n-s_{1}, \ldots, n-s_{n}\right)$ is a parking function as defined in Stanley [7,8] i.e., a sequence of $n$ integers between 0 and $n-1$ at most $i$ of which are $\geq n-i$ for all $1 \leq i \leq n$.

The area of the major sequence $S=\left(s_{1}, \ldots, s_{n}\right)$ is defined as

$$
a(S)=\sum_{i=1}^{n} s_{i}-\left(\begin{array}{c}
n+1 \\
2
\end{array}\right) .
$$


It is non-negative and is the area between the lattice path and the main diagonal. The area of the sequence in Figure 1 s 8 , as illustrated by the shaded boxes. We denote by $\mathcal{M}_{n}(k)$ the set of major sequences of length $n$ and area $k$, and define the area enumerator for major sequences of length $n$ as

$$
M_{n}(t)=\sum_{S} t^{a(S)}
$$

where the sum is over all major sequences of length $n$.

To define external activity, we consider a complete graph $K$ on $\{0, \ldots, n\}$. We order its edges lexicographically, i.e., edge $i j$ with $i<j$ is smaller than edge $k l$ with $k<l$ iff $(i<k)$ or $(i=k, j<l)$. Let $T$ be a spanning tree of $K$. An edge of $K-T$ is called externally active for $T$ if it is the smallest edge in the unique cycle that it closes with edges of $T$. For example, the tree $T$ in Figure 2 has exactly 8 externally active edges, namely 01, 02, 03, 04, 05, 23, 2645.

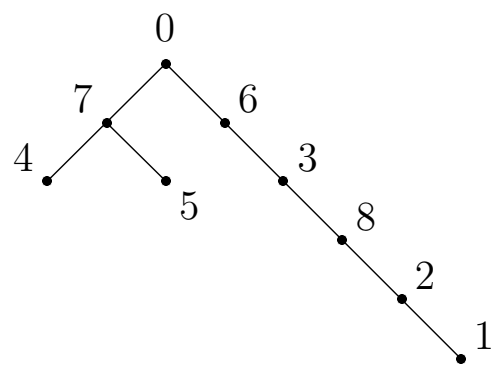

$T$

Figure 2: A tree with external activity 8 and 10 inversions.

The external activity $e(T)$ is the number of externally active edges for $T$. We denote by $\mathcal{E}_{n+1}(k)$ the set of trees on the vertex set $\{0, \ldots, n\}$ with external activity $k$, and define the external activity enumerator for trees on $\{0, \ldots, n\}$ as

$$
E_{n+1}(t)=\sum_{T} t^{e(T)}
$$

where the sum is over all trees on $\{0, \ldots, n\}$. We remark that $E_{n+1}(t)$ is the Tutte polynomial of $K$ evaluated at $(1, t)$. See Gessel [3] and Gessel and Sagan [4] for many properties of the Tutte polynomial and further references.

If $T$ is a tree on $\{0, \ldots, n\}$, an inversion of $T$ is a pair $(i, j)$ such that $1 \leq j<i \leq n$ and $i$ lies on the path from 0 to $j$ in $T$. For example, the tree $T$ in Figure 2 has exactly 
10 inversions, namely $(2,1),(3,1),(3,2),(6,1),(6,2),(6,3),(7,4),(7,5),(8,1),(8,2)$. We denote by $i(T)$ the number of inversions of $T$. We also denote by $\mathcal{I}_{n+1}(k)$ the set of trees on $\{0, \ldots, n\}$ with $k$ inversions and define the inversion enumerator for trees on $\{0, \ldots, n\}$ as

$$
I_{n+1}(t)=\sum_{T} t^{i(T)},
$$

where the sum is over all trees on $\{0, \ldots, n\}$.

Björner discovered that

$$
I_{n+1}(t)=E_{n+1}(t),
$$

using his results on shellability and homology in matroids as well as a result of Gessel and Wang [5] (see Exercise $7.7(\mathrm{c})$, page 271 of [2]). Beissinger [1] proved (1) by providing a bijection from $\mathcal{I}_{n+1}(k)$ to $\mathcal{E}_{n+1}(k)$.

Kreweras [6] showed that

$$
M_{n}(t)=I_{n+1}(t),
$$

and gave a bijection from $\mathcal{M}_{n}(k)$ to $\mathcal{I}_{n+1}(k)$.

An immediate consequence of (1) and (2) is

$$
M_{n}(t)=E_{n+1}(t) .
$$

We prove (3) by presenting a direct bijection from $\mathcal{M}_{n}(k)$ to $\mathcal{E}_{n+1}(k)$. It uses the decomposition of major sequences that Kreweras used, but because it avoids inversions, it is simpler than both the bijections of Kreweras and of Beissinger.

We reproduce Kreweras' decomposition below for completeness. In preparation for it we note that, by definition, if $\left(s_{1}, \ldots, s_{n}\right)$ is a major sequence and we increase $s_{n}$ (or any other $s_{i}$ ) to a larger integer not exceeding $n$, the new sequence is still major. Conversely, if we repeatedly decrease $s_{n}$ by 1 , eventually the sequence will no longer be major. We denote by $s_{n}^{*}$ the smallest integer $s$ such that $\left(s_{1}, \ldots, s_{n-1}, s\right)$ is still a major sequence, and call $\left(s_{1}, \ldots, s_{n-1}, s_{n}^{*}\right)$ the reduced form of $\left(s_{1}, \ldots, s_{n}\right)$. For example, for the major sequence $(7,8,8,3,3,5,3,7)$ that we saw in Figure $1, s_{8}^{*}=4$, and the nondecreasing rearrangement of its reduced form is shown in Figure 3.

If $x=\left(x_{1}, \ldots, x_{n}\right)$ is an integer sequence, we denote its nondecreasing rearrangement by $\operatorname{sort}(x)=\operatorname{sort}\left(x_{1}, \ldots, x_{n}\right)$. For any integer $c$, we denote the sequence $\left(x_{1}+c, \ldots, x_{n}+c\right)$ by $x+c$.

The Decomposition Lemma Let $\left(s_{1}, \ldots, s_{n}\right)$ be a major sequence and let

$$
\left(z_{1}, \ldots, z_{n}\right)=\operatorname{sort}\left(s_{1}, \ldots, s_{n-1}, s_{n}^{*}\right)
$$

be the nondecreasing rearrangement of its reduced form. Then 




Figure 3: The nondecreasing rearrangement of $(7,8,8,3,3,5,3,4)$, the reduced form of the major sequence $(7,8,8,3,3,5,3,7)$ of Figure 1 .

1. There exists a unique $l$ satisfying $z_{l}=s_{n}^{*}$, namely $l=s_{n}^{*}$;

2. $z_{l-1}<z_{l}<z_{l+1}$ (where $z_{0}$ and $z_{n+1}$ are understood to be 0 and $n+1$, respectively);

3. $\left(z_{1}, \ldots, z_{l-1}\right)$ and $\left(z_{l+1}, \ldots, z_{n}\right)-l$ are major sequences;

4. $a\left(s_{1}, \ldots, s_{n-1}, s_{n}^{*}\right)=a\left(z_{1}, \ldots, z_{l-1}\right)+a\left(\left(z_{l+1}, \ldots, z_{n}\right)-l\right)$, and consequently $a\left(s_{1}, \ldots, s_{n}\right)=a\left(z_{1}, \ldots, z_{l-1}\right)+a\left(\left(z_{l+1}, \ldots, z_{n}\right)-l\right)+\left(s_{n}-s_{n}^{*}\right)$.

Proof. 1. Clearly $z_{l}=s_{n}^{*}$ for some $l$. Since $\left(z_{1}, \ldots, z_{n}\right)$ is a major sequence, we have $z_{l} \geq l$ and therefore $s_{n}^{*} \geq l$. But if this inequality is strict, then $\left(z_{1}, \ldots, z_{l-1}, z_{l}-\right.$ $\left.1, z_{l+1}, \ldots, z_{n}\right)$, which is a rearrangement of $\left(s_{1}, \ldots, s_{n-1}, s_{n}^{*}-1\right)$, would still be a major sequence, contrary to the definition of $s_{n}^{*}$. Hence $s_{n}^{*}=l$.

2. This follows immediately from 1) above, for if $z_{l \pm 1}=z_{l}$, then $s_{n}^{*}$ would equal both $l$ and $l \pm 1$.

3. This also follows from 1) above, since the lattice path returns to the main diagonal at $(l, l)$, and is also easy to verify algebraically using 2$)$.

4. This too follows from the fact that the lattice path returns to the main diagonal at $(l, l)$, and is verifiable by an easy calculation. 


\section{The Bijection}

We now construct a mapping $f$ from the set of major sequences to the set of labeled trees that maps $\mathcal{M}_{n}(k)$ to $\mathcal{E}_{n+1}(k)$ as follows.

1. Given a major sequence $S=\left(s_{1}, \ldots, s_{n}\right)$, find its reduced form

$$
\left(s_{1}, \ldots, s_{n-1}, s_{n}^{*}\right)
$$

2. Set

$$
E_{1}=\left\{i: 1 \leq i \leq n-1, s_{i}<s_{n}^{*}\right\}, \quad E_{2}=\left\{i: 1 \leq i \leq n-1, s_{i}>s_{n}^{*}\right\} .
$$

By Part 2 of the Decomposition Lemma, $E_{1}$ and $E_{2}$ partition $\{1, \ldots, n-1\}$. Set

$$
S_{1}=\left(s_{i}: i \in E_{1}\right), \quad S_{2}=\left(s_{i}: i \in E_{2}\right)-s_{n}^{*} .
$$

By part 3 of the Decomposition Lemma, $S_{1}$ and $S_{2}$ are major sequences of length $l-1$ and $n-l$, respectively, with $l=s_{n}^{*}$ as in the lemma. Recursively obtain the trees $T_{1}=f\left(S_{1}\right)$ and $T_{2}=f\left(S_{2}\right)$ on $\{0, \ldots, l-1\}$ and $\{0, \ldots, n-l\}$ respectively, with $e\left(T_{1}\right)=a\left(S_{1}\right)$ and $e\left(T_{2}\right)=a\left(S_{2}\right)$, and thus by Part 4 of the Decomposition Lemma

$$
e\left(T_{1}\right)+e\left(T_{2}\right)+\left(s_{n}-s_{n}^{*}\right)=a(S) .
$$

3. Relabel the vertices of $T_{1}-\{0\}$ with the elements of $E_{1}$, preserving their order, which gives the tree $T_{1}^{\prime}$ with $e\left(T_{1}^{\prime}\right)=e\left(T_{1}\right)$. Relabel the vertices of $T_{2}$ with the elements of $E_{2} \cup\{n\}$, preserving their order, which gives the tree $T_{2}^{\prime}$ with $e\left(T_{2}^{\prime}\right)=e\left(T_{2}\right)$.

4. Let $r$ be the $\left(s_{n}-s_{n}^{*}+1\right.$ )-st smallest vertex in $T_{2}^{\prime}$ (this vertex exists since $\left.1 \leq s_{n}-s_{n}^{*}+1=s_{n}-l+1 \leq n-l+1\right)$. Connect vertex 0 of $T_{1}^{\prime}$ with vertex $r$ of $T_{2}^{\prime}$ to obtain the tree $T=f(S)$ on $\{0, \ldots, n\}$.

5. We have

$$
e(T)=e\left(T_{1}^{\prime}\right)+e\left(T_{2}^{\prime}\right)+\left(s_{n}-s_{n}^{*}\right)
$$

because the only externally active edges of $T$ between $T_{1}^{\prime}$ and $T_{2}^{\prime}$ are the $s_{n}-s_{n}^{*}$ edges joining 0 with the vertices of $T_{2}^{\prime}$ smaller than $r$. Therefore

$$
e(T)=a(S)
$$

as required. 
For example, given our major sequence $(7,8,8,3,3,5,3,7)$, we find that $s_{8}^{*}=4$, $S_{1}=(3,3,3), S_{2}=(3,4,4,1)$ and $E_{1}=\{4,5,7\}, E_{2}=\{1,2,3,6\}$. Note that in Figure 3, $\operatorname{sort}\left(S_{1}\right)$ is shown to the left of the bar of height $s_{n}^{*}=4$ and $\operatorname{sort}\left(S_{2}\right)$ is shown above the dotted line to the right of that bar. Note also that $E_{1}$ and $E_{2}$ are the sets of positions of those elements of $S$ that are used to form $S_{1}$ and $S_{2}$, respectively. The trees $T_{1}$ and $T_{2}$, obtained recursively, are shown in Figure 4.
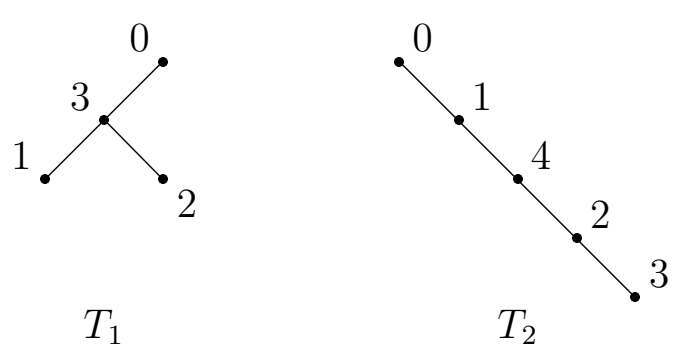

Figure 4: Trees $T_{1}$ and $T_{2}$ obtained in Step 2 of the bijection.

The relabelings $T_{1}^{\prime}$ and $T_{2}^{\prime}$ obtained in Step 3 are shown in Figure 5. The vertex $r$ in
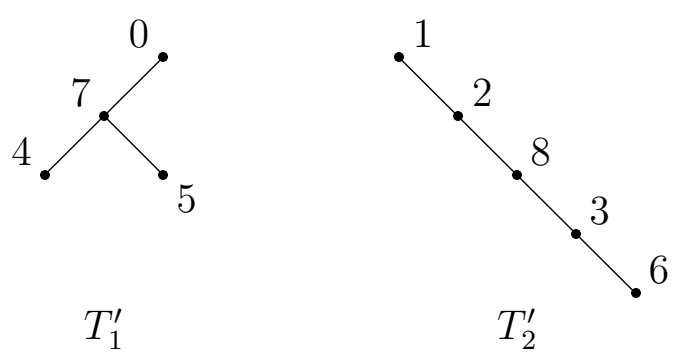

Figure 5: Trees $T_{1}^{\prime}$ and $T_{2}^{\prime}$ obtained in Step 3 of the bijection.

Step 4 is the fourth smallest vertex in $T_{2}^{\prime}$, namely $r=6$, and the final tree $T$ is the one shown in Figure 2 .

We now present the inverse mapping $f^{-1}$ from trees to major sequences.

1. Given a tree $T$ on $\{0, \ldots, n\}$, let $0 r$ be the first edge along the path from 0 to $n$ in $T$. Deleting this edge leaves two subtrees: $T_{1}^{\prime}$ with $l$ vertices including 0 , and $T_{2}^{\prime}$ with $n+1-l$ vertices including $r$.

2. Relabel the vertices of $T_{1}^{\prime}$ as $0, \ldots, l-1$, preserving their order, to obtain the tree $T_{1}$. Recursively obtain the major sequence

$$
S_{1}=\left(a_{1}, \ldots, a_{l-1}\right)=f^{-1}\left(T_{1}\right) .
$$


THE ELECTRONiC JOURNAL OF COMBINATORICS 4 (No. 2) (1997), \#R4

This $S_{1}$ will be a subsequence of the sequence $S$ that we are constructing. Specifically, put the elements of $S_{1}$ in order into the positions of $S$ indexed by the vertices of $T_{1}^{\prime}-\{0\}$, i.e., if $i$ is the $j$-th smallest vertex in $T_{1}^{\prime}-\{0\}$, set $s_{i}=a_{j}$. Relabel the vertices of $T_{2}^{\prime}$ as $0, \ldots, n-l$, preserving their order, to obtain the tree $T_{2}$. Recursively obtain the major sequence

$$
S_{2}=\left(b_{1}, \ldots, b_{n-l}\right)=f^{-1}\left(T_{2}\right) .
$$

Put the elements of $S_{2}+l$ in order into the positions of $S$ indexed by the vertices of $T_{2}^{\prime}-\{n\}$, i.e., if $i$ is the $j$-th smallest vertex in $T_{2}^{\prime}-\{n\}$, set $s_{i}=b_{j}+l$.

3. We assert that $\left(s_{1}, \ldots, s_{n-1}, l\right)$ is a major sequence. Indeed, since the elements of $S_{1}$ are smaller than $l$ and the elements of $S_{2}+l$ are larger than $l$, we have

$$
\operatorname{sort}\left(s_{1}, \ldots, s_{n-1}, l\right)=\left(z_{1}, \ldots, z_{l-1}, l, z_{l+1}, \ldots, z_{n}\right),
$$

where $\left(z_{1}, \ldots, z_{l-1}\right)=\operatorname{sort}\left(S_{1}\right)$ and $\left(z_{l+1}, \ldots, z_{n}\right)=\operatorname{sort}\left(S_{2}+l\right)$. Hence $z_{i} \geq i$ for $1 \leq i \leq l-1$ and $z_{l+i} \geq l+i$ for $1 \leq i \leq n-l$, and furthermore $z_{n} \leq(n-l)+l=n$, proving the assertion.

4. Put $s_{n}=l+q$, where $q$ is the number of vertices of $T_{2}^{\prime}$ smaller than $r$. We have $s_{n} \leq$ (number of vertices of $\left.T_{1}^{\prime}\right)+\left(\right.$ number of vertices of $\left.T_{2}^{\prime}-1\right)=n$.

Since we have obtained $S=\left(s_{1}, \ldots, s_{n}\right)$ from the major sequence $\left(s_{1}, \ldots, s_{n-1}, l\right)$ by increasing its last component, but not above $n, S$ is a major sequence.

5. Using induction and the familiar arguments, we obtain

$$
\begin{aligned}
a(S) & =a\left(z_{1}, \ldots, z_{l-1}\right)+a\left(\left(z_{l+1}, \ldots, z_{n}\right)-l\right)+q \\
& =a\left(S_{1}\right)+a\left(S_{2}\right)+q \\
& =e\left(T_{1}\right)+e\left(T_{2}\right)+q \\
& =e\left(T_{1}^{\prime}\right)+e\left(T_{2}^{\prime}\right)+q \\
& =e(T) .
\end{aligned}
$$

Furthermore, the major sequence $S$ just constructed satisfies $s_{n}^{*}=l$, as can be seen from the argument in 3 above. From this it follows easily that $f(S)=T$, and therefore we have indeed inverted $f$, so $f$ is a bijection.

We remark that in mapping major sequences to trees, Kreweras' algorithm and ours use the same decomposition, but obtain different trees. The algorithms differ, first, in how vertex $r$ is chosen and, second, in the fact that Kreweras' algorithm permutes a subset of the labels of $T_{2}^{\prime}$ (to obtain the correct number of inversions) and ours does not have to. A similar permutation of a subset of labels also occurs in Beissinger's algorithm. 
THE ELECTRONiC JOURNAL OF COMBINATORICS 4 (No. 2) (1997), \#R4

\section{Acknowledgement}

We thank Richard Stanley for encouraging us to work on this problem, and for providing us with valuable references.

\section{References}

[1] J. S. Beissinger. On external activity and inversions in trees. J. Combin. Theoy Ser. B, 33(1):87-92, 1982.

[2] A. Björner. The homology and shellability of matroids and geometric lattices. In N. White, editor, Matroid Applications, chapter 7, pages 226-283. Cambridge University Press, Cambridge, 1991.

[3] I. M. Gessel. Enumerative applications of a decomposition for graphs and digraphs. Discrete Math., 139:257-271, 1995.

[4] I. M. Gessel and B. E. Sagan. The tutte polynomial of a graph, depth-first search, and simplicial complex partitions. Electronic J. of Combinatorics, 3(2):\#R9, 1996.

[5] I. M. Gessel and D.-L. Wang. Depth-first search as a combinatorial correspondence. J. of Combin. Theory Ser. A, 26:308-313, 1979.

[6] G. Kreweras. Une famille de polynômes ayant plusiers propriétés énumeratives. Periodica Math. Hung., 11(4):309-320, 1980.

[7] R. P. Stanley. Hyperplane arrangements, interval orders, and trees. Proc. Natl. Acad. Sci. USA, Mathematics, 93:2620-2625, March 1996.

[8] R. P. Stanley. Hyperplane arrangements, parking functions and tree inversions. Preprint, May 1996. 